\title{
Prevenção da doença pneumocócica no Brasil
}

\author{
Prevention of pneumococcal disease in Brazil
}

\author{
Gabriel Oselka*
}

\begin{abstract}
Nesta edição do Jornal de Pediatria, NascimentoCarvalho e cols. ${ }^{1}$ apresentam o resultado de estudo que avalia as cepas invasivas de pneumococo, isoladas de pacientes com menos de 20 anos de idade, e Lucarevschi e cols. ${ }^{2}$ apresentam dados sobre a colonização de orofaringe por Streptococcus pneumoniae em crianças de creche municipais de Taubaté, SP. Nos dois estudos, é apresentada correlação entre os sorotipos isolados e os representados na vacina conjugada heptavalente contra o pneumococo (VCP7V), recentemente licenciada no Brasil. A concordância entre os sorotipos presentes na vacina e os isolados nos dois estudos foi de $67,2 \%$ e $63 \%$,
\end{abstract} respectivamente.

Há muitos anos, é disponível no Brasil uma vacina constituída por uma mistura de polissacárides de 23 diferentes sorotipos $(1,2,3,4,5,6 \mathrm{~B}, 7 \mathrm{~F}, 8,9 \mathrm{~N}, 9 \mathrm{~V}, 10 \mathrm{~A}$,

\section{Veja artigos relacionados nas páginas 209 e 215}

A solução para esse problema foi inspirada nas vacinas conjugadas contra o Hib. A conjugação de polissacárides a proteínas muda a natureza da resposta aos PSs, de Tindepentente para T-dependente. Esse complexo antigênico estimula resposta com linfócitos T-helper, o que significa excelente resposta em lactentes jovens e sólida memória imunológica.

No Brasil, existe licenciada uma vacina conjugada heptavalente contra o pneumococo, que inclui os polissacárides capsulares de sete sorotipos de pneumococos $(4,6 \mathrm{~B}$, $9 \mathrm{~V}, 14,18 \mathrm{C}, 19 \mathrm{Fe} 23 \mathrm{~F}$ ), conjugados individualmente a uma variante não tóxica da toxina diftérica, CRM 197 (CRM - cross-reacting-material). Contém fosfato de alumínio como adjuvante, sendo destituída de timerosal ou outro conservante.

Problemas de natureza técnica (bem 11A, 12F, 14, 15B, 17F, 18C, 19F, 19A, 20, 22F, 23F e $33 \mathrm{~F}$ ), que representam $85-90 \%$ dos sorotipos responsáveis por doença invasiva nos EUA, e pouco mais de $80 \%$ no Brasil.

Entretanto, a vacina 23-valente, se, por um lado, tem a vantagem de espectro amplo, apresenta a grande desvantagem (característica das vacinas polissacarídicas em geral) de estimular resposta imune independente de células $T$, ou seja, são estimulados apenas linfócitos B e não linfócitos T. Por isso, a resposta imune aos antígenos T-indendentes é de curta duração, não havendo, também, indução de memória imunológica. Além disso, e, principalmente, crianças com menos de dois anos de idade não respondem adequadamente aos antígenos T-independentes, ao contrário do que ocorre com os T-dependentes (por ex., proteínas), que induzem resposta imune logo após o nascimento, de longa duração e com memória. No caso da vacina polissacarídica contra os pneumococos, isso significa resposta deficiente exatamente na faixa etária de maior risco em crianças.

\footnotetext{
* Professor Associado, Departamento de Pediatria da Faculdade de Medi-
} cina da Universidade de São Paulo. como, eventualmente, de eventos adversos) impedem, no momento, a produção de vacinas conjugadas com 23 sorotipos. Estão sendo estudadas vacinas 9 e 11-valentes (ambas contêm, além dos presentes na VCP7V, os sorotipos 1 e 5); entretanto, é improvável que venham a ser licenciadas nos próximos dois a três anos.

$\mathrm{O}$ número menor de sorotipos na vacina levanta $\mathrm{o}$ problema da cobertura oferecida para as infecções pneumocócicas, especialmente para as crianças com menos de seis anos de idade, que são o grupo etário para o qual hoje a vacinaé recomendada ${ }^{3,4}$. Os sorotipos incluídos na VCP7V foram responsáveis por $86 \%$ das bacteremias, $83 \%$ da meningites e $65 \%$ das OMAs pneumocócicas em crianças com menos de seis anos de idade, no período 1978-1994, nos Estados Unidos.

Graças aos excelentes dados obtidos com a investigação laboratorial de cepas invasivas de pneumococo no âmbito do projeto SIREVA (Vigilância Epidemiologicas de Streptococcus pneumoniae na América Latina), conhecemos bem a prevalência dos sorotipos de pneumococos isolados de doenças invasivas (e sua resistência à penicilina e a outros antimicrobianos) em diferentes regiões geográficas do Brasil. 
De acordo com resultados apresentados pela principal investigadora no Brasil, M. Cristina Brandileone, os 15 sorotipos prevalentes em 1.069 crianças com menos de seis anos de idade, no período de 1993 a 1998, com doença invasiva (principalmente meningite e pneumonia), foram os seguintes (percentuais de participação entre parênteses): 14 $(24,7) ; 1(10,3) ; 6 \mathrm{~B}(10,0) ; 18 \mathrm{C}(7,9) ; 5(6,5) ; 6 \mathrm{~A}(5,4) ; 23 \mathrm{~F}$ $(5,2) ; 19 \mathrm{~F}(4,5) ; 9 \mathrm{~V}(4,2) ; 19 \mathrm{~A}(3,2) ; 3(2,3) ; 4(1,6) ; 10 \mathrm{~A}$ $(1,6) ; 8(1,4) ; 7 \mathrm{~F}(0,7)$. Outros sorotipos respondem por apenas $10,5 \%$ dos casos 5 .

Comparando esses dados com os encontrados nos Estados Unidos (EUA), constata-se importante diferença: a cobertura oferecida pela vacina 7 -valente seria de $63,5 \%$, devido à importante participação dos sorotipos 1 e 5 , que não são relevantes nos EUA, não tendo sido, por isso, incluídos na vacina.

Quando se analisam os dados relativos à resistência dos pneumococos à penicilina (que vem aumentando de forma preocupante nos últimos anos), verificamos que, no município de São Paulo, 2,4\% e 24, 4\% das cepas isoladas em menores de seis anos de idade apresentavam alta resistência e resistência intermediária à penicilina, respectivamente. Entretanto, cerca de $90 \%$ das amostras de sorotipos resistentes seriam cobertos pela VCP7V, já que a resistência concentrou-se nos sorotipos 14 ( $41 \%$ do total), $6 \mathrm{~B}(21,5 \%)$, 19A $(8,3 \%)$ e $19 \mathrm{~F}(3,0 \%)$. Os sorotipos 1 e 5 são muito raramente resistentes à penicilina 5 .

Em trabalho recente, Brandileone e cols. apresentam dados atualizados do projeto SIREVA, estratificados por faixa etária para meningite, pneumonia e outras doenças pneumocócicas invasivas. A cobertura estimada para a vacina heptavalente em crianças de sete meses a cinco anos de idade foi de $58,2 \%$ para todos as doenças invasivas; a inclusão dos sorotipos 1 e 5 aumentaria em $15 \%$ o potencial de cobertura (73\%). A cobertura mais elevada para VCP7V, nos casos de pneumonia, foi observada na faixa etária de sete meses a dois anos de idade $(70 \%)$; nesta idade, a cobertura para meningite seria de $61 \%$. É importante salientar que a possível cobertura reduz-se para $29,2 \%$ e $51,7 \%$, respectivamente, nas crianças entre dois e cinco anos de idade ${ }^{6}$.

Embora os resultados do estudo de Nascimento-Carvalho e cols. sejam semelhantes aos de Brandileone e cols. quanto à cobertura da VPC7V para doenças invasivas, há notáveis diferenças quando os sorotipos isolados são comparados, chamando a atenção a raridade de isolamento do sorotipo $1(1,4 \%)$ em Salvador.

Ficam evidentes, uma vez mais, as diferenças regionais na distribuição de sorotipos no Brasil, o que ressalta a importância de estudos como os de Nascimento-Carvalho e cols. e Lucarevschi e cols.

A efetividade da VCP7V verificada nos Estados Unidos não deve, pelas razões apresentadas, repetir-se no Brasil. Naquele país, o impacto da introdução da VC7V na rotina foi avaliada na população que se utiliza dos serviços do Grupo Kaiser Permanente (GKP, um grupo de assistência médica sediado na Califórnia). A vacinação rotineira das crianças até cinco anos de idade começou em abril de 2000; nessa ocasião, o GKP atendia 211.565 crianças menores de cinco anos de idade. A incidência de doença pneumocócica invasiva causada por sorotipos presentes na vacina (de 1995 até o início da vacinação de rotina) variou de 51,5 a 98,1 casos por 100.000 pessoas-ano, em crianças com menos de um ano de idade, e caiu para 9,35 após a introdução da vacina (redução de 87,3\%); em menores de dois anos de idade, a redução foi de $58,1 \%$, e em menores de cinco anos de idade, $62,4 \%{ }^{7}$.

Em todas as faixas etárias citadas a magnitude da redução foi substancialmente maior que a percentagem de crianças que haviam recebido uma ou mais doses da vacina, ou o esquema completo para a idade. Esses dados sugerem que a vacina pode determinar imunidade coletiva (herd immunity), ou seja, proteção indireta para as crianças não vacinadas, além dos altos níveis de proteção verificados para as crianças vacinadas. Além disso, não se verificou aumento correspondente no risco de doença invasiva causada por sorotipos não presentes na vacina ${ }^{7}$.

A VCP7V representa importante progresso na profilaxia das infecções pneumocócicas de crianças. Sua atual composição, sem a presença dos sorotipos 1 e 5 , não é a ideal para o Brasil; além disso, seu elevado custo limita a utilização inicial entre nós, à semelhança das vacinas contra o Hib e contra a hepatite B, em passado recente, e das vacinas contra a varicela, a hepatite $\mathrm{A}$ e o meningococo $\mathrm{C}$ (conjugada), atualmente. Acreditamos, porém, que, sempre que possível, seu uso deve ser encorajado.

\section{Referências bibliográficas}

1. Nascimento-Carvalho CM, Freitas-Souza LS, Moreno-Carvalho OA, Alves NN, Caldas RM, Barberino MG, et al. Cepas invasivas de pneumococo isoladas de crianças e adolescentes em Salvador. J Pediatr (Rio J) 2003;79:209-14.

2. Lucarevschi BR, Baldacci ER, Bricks LF, Bertoli CJ, Teixeira LM, Mendes CMF, et al. Colonização de orofaringe por Streptococcus pneumoniae em crianças de creches municipais de Taubaté-SP: correlação entre os principais sorotipos e a vacina pneumocócica conjugada heptavalente. J Pediatr (Rio J) 2003;79:215-20.

3. American Academy of Pediatrics, Committee on Infectious Diseases. Policy statement: recommendations for the prevention of pneumococcal infections, including the use of pneumococcal conjugate vaccine (Prevnar), pneumococcal polysaccharide vaccine, and antibiotic prophylaxis. Pediatrics 2000;106:362-6.

4. Centers for Disease Control and Prevention. Preventing pneumococcal disease among infants and young children: recommendations of the Advisory Committee on Immunization Practices (ACIP). MMWR 2000;49(RR-9):1-35.

5. Brandileone MCC. Distribuição de sorotipos, resistência antimicrobiana e perfil molecular de Streptococcus pneumoniae isolado de doença invasiva no Brasil: 1993 a 1998 [tese de doutorado]. São Paulo: Escola Paulista de Medicina; 1999.

6. Brandileone MCC, Andrade ALS, Di Fabio JL, Guerra. LLS, Austrian R. Appropriateness of a pneumococcal conjugate vaccine in Brazil: potential impact of age and clinical diagnosis, with emphasis on meningitis. J Infect Dis 2003;187:1206-12.

7. Black SB, Shinefield HR, Hansen J, Elvin L, Laufer D, Malinoski F. Postlicensure evaluation of the effectiveness of seven valent pneumococcal conjugate vaccine. Pediatr Infect Dis J 2001;20: 1105-7. 